\title{
THE INTERNAL BIAS FIELD OF FERROELECTRIC $\left(\mathrm{CH}_{3} \mathrm{NH}_{3}\right)_{5} \mathrm{Bi}_{2} \mathrm{Br}_{11}$
}

\author{
H. PyKacz, J. Mróz \\ Institute of Physics, Technical University of Wrocław \\ Wybrzeze Wyspiańskiego 27, 50-370 Wrocław, Poland \\ AND R. J AKUBAS \\ Institute of Chemistry, University of Wroclaw \\ F. Joliot-Curie 14, 50-383 Wroclaw, Poland
}

(Received December 17, 1991; in final form May 7, 1992)

\begin{abstract}
Spontaneous polarization of $\left(\mathrm{CH}_{3} \mathrm{NH}_{3}\right)_{5} \mathrm{Bi}_{2} \mathrm{Br}_{11}$ crystals has been measured and the ferroelectric hysteresis loop has been studied. The observed time changes in the polarization have been ascribed to the change in the internal bias field of the crystal. Measurement results have also been discussed for the lower phase transition $\left(T_{1 \mathrm{I}}=77 \mathrm{~K}\right)$.
\end{abstract}

PACS numbers: $77.70 .+a$

\section{Introduction}

The alkylammonium halogenobismuthate, $\left(\mathrm{CH}_{3} \mathrm{NH}_{3}\right)_{5} \mathrm{Bi}_{2} \mathrm{Br}_{11}$, crystallizes at room temperature in orthorhombic symmetry space group, $P c a 2_{1}$, and undergoes a second-order phase transition at $T_{\mathrm{c}}=312 \mathrm{~K}$ to ferroelectric phase [1-2]. It is assumed that the phase transition is connected with the ordering of $\mathrm{CH}_{3} \mathrm{NH}_{3}{ }^{+}$ cations. Dielectric anomaly [1], [3-6], differential scanning calorimeter (DSC) [7], birefringence [8], and dilatometric [9] studies confirm the continuous character of the ferro-paraelectric phase transition. The second much smaller dielectric anomaly along the $c$-direction at $77 \mathrm{~K}$ was interpreted as a phase transition [4], [10].

The published results show, however, some discrepances. The extremal values of the ratio $C_{\mathrm{p}} / C_{\mathrm{f}}$ of the Curie constants in the para- and ferroelectric phases are equal to 3.6 [1] and 5.6 [4]. Spontaneous polarization in units of $10^{-2} \mathrm{C} / \mathrm{m}^{2}$ below the phase transition temperature $T_{\mathrm{c}}$ ranges from $0.7(\Delta T=27 \mathrm{~K})$ [1] to $1.75(\Delta T=12 \mathrm{~K})[10]$. Character of some phenomena observed in that crystal is not typical, namely high $C_{\mathrm{p}} / C_{\mathrm{f}}$, anomalous electric permittivity along the $a$ and $b$-directions [1] and discrepancy in the values of the spontaneous polarization for crystals biased with different electric fields [6]. The reasons of that can be 
numerous; the one is due to crystal imperfections caused by the crystal defects. The aim of this work is to determine chemical defect impact (especially the presence of $\mathrm{Br}_{2}$ in the lattice) on the dielectric response of $\left(\mathrm{CH}_{3} \mathrm{NH}_{3}\right)_{5} \mathrm{Bi}_{2} \mathrm{Br}_{11}$ in the vicinity of the phase transition. From the published papers it is known that the crystals were grown either from highly [5] or from slightly [1] HBr-rich solutions.

\section{Experimental}

The crystals were grown as described in Ref. [1] with a high excess of $40 \%$ HBr. A few crystals were grown from the same solution. The dielectric permittivity was measured at $1 \mathrm{kHz}$. The pyroelectric coefficient was measured by the short-circuit current method at the constant rate of $1.3 \times 10^{-2} \mathrm{~K} / \mathrm{s}$ on heating. Previously electrical poling was achieved by cooling a crystal from the para- into the ferroelectric phase in the applied electric field of $300 \mathrm{kV} / \mathrm{m}$. The spontaneous polarization was measured with the Sawyer-Tower circuit at $50 \mathrm{~Hz}$, by the pyroelectric charge method with an electrometer, and also was calculated by intergration of the pyroelecric coefficient.

\section{Results}

Electric permittivity was measured close to the phase transition temperature $T_{\mathrm{c}}$. The constants determined from the Curie-Weiss law are as folows: $C_{\mathrm{p}}=2800 \mathrm{~K}, C_{\mathrm{f}}=560 \mathrm{~K}$, and $C_{\mathrm{p}} / C_{\mathrm{f}}=5$. The phase-transition temperature determined from dielectric and pyroelectric measurements was $309 \mathrm{~K}$, being lower by $3 \mathrm{~K}$ than that determined by Jakubas [1]. Observations of a ferroelectric hysteresis as a function of electric field and time rendered it possible to conclude certain rules. The hysteresis loop shape and the electric polarization were found to be determined by the rise time of the applied electric field. When the electric field increases with time very slowly, the hysteresis for fields below the threshold ( $E<E_{1}$, cf. Fig. 1a) is not observed and the $P(E)$ relation is linear. The pyroelectric measurements for an as-grown sample indicate the non-zero unipolarity. For fields $E_{1}<E<E_{2}$ there is a hysteresis loop and the polarization rapidly increases with field. The field $E_{1}$ being equal to the threshold is called the threshold field. At the field of $E_{2}$ the spontaneous polarization assumes to be constant and the saturation time lasts a few to several seconds.

When the electric field is decreasing back towards $E_{1}$ and below, the spontaneous polarization does not change at the beginning, but further on both the polarization and the coercive field $E_{\mathrm{c}}$ are decreasing upon decrease of the electric field and more rapidly upon approaching of $E_{3}$ (cf. Fig. 1a). At the field $E_{3}>E_{\mathrm{c}}$ the hysteresis disappears. The results were repeatable when breaks between the measurements lasted at least a few hundreds seconds. For shorter breaks the thresholds field was lower. A quite different picture of the hysteresis is achieved when the field is step-like. At the field of $E_{1}+\Delta E$ there is a single (cf. Fig. 1b) or double (cf. Fig. 1c) hysteresis loop at the ends of the $P(E)$ relation with an internal bias field $E_{\mathrm{b}}$ depending upon the unipolarity. The $E_{\mathrm{b}}$ field decreases to zero within 8-10 seconds, and at last a single hysteresis remains (cf. Fig. 1c, dashed line). For 
the repeated field rise the double hysteresis can occur again with the internal bias field of $E_{\mathrm{b} 2}>E_{\mathrm{b} 1}$ which decreases with time. The cycle can be repeated even a couple of times.

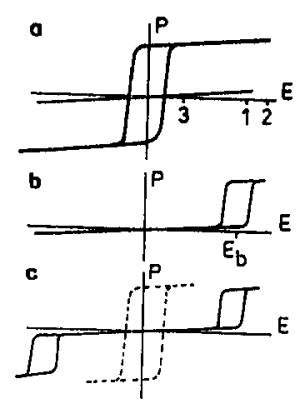

Fig. 1. Sketch of ferroelectric hysteresis loops with marked electric field. (a) $E_{1}=E_{\mathrm{p}}$ is the threshold which is necessary to form the hysteresis loop. In the field $E_{2}$ and higher a rise of the loop to a constant hight proceeds quickly. In the field $E_{3}$ the hysteresis loop disappears. After switching the field $E_{2}$ on one observes a single or double loop ((b) and (c)) which at last passes to a single one. Such pictures on a screen were observed at temperatures a few degree below the phase transition temperature $T_{\mathrm{c}}$.

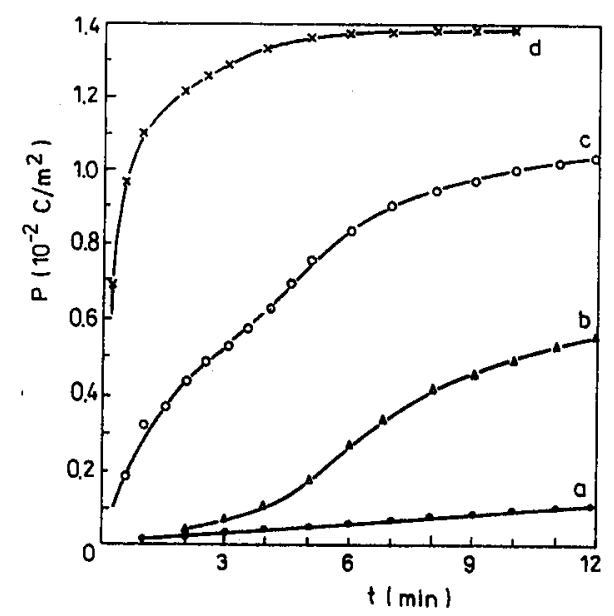

Fig. 2. Temporary changes of the electric polarizations at the temperature $8 \mathrm{~K}$ below $T_{\mathrm{c}}$ obtained from the hysteresis loop. The loop appears at the threshold field $E_{\mathrm{p}}=$ $1150 \mathrm{kV} / \mathrm{m}$. The amplitude of the applied field $\mathrm{E}$ and the coercive field $E_{\mathrm{c}}$ in units of $\mathrm{kV} / \mathrm{m}$ are the following : a) 1190,115 ; b) 1220,250 ; ) 1250,270 ; d) 1300, 280. The loop disappears at $E_{0}=480 \mathrm{kV} / \mathrm{m}$.

Figure 2 shows the time dependence of the electric polarization for various electric bias fields at $\left(T_{\mathrm{c}}-T\right)=8 \mathrm{~K}$. At the threshold field $E_{\mathrm{p}}=1150 \mathrm{kV} / \mathrm{m}$ and lower, there is no hysteresis $\left(E_{\mathrm{p}} \equiv E_{1}\right)$. At the field of $1190 \mathrm{kV} / \mathrm{m}$ the electric 
polarization increases linearly with time (cf. curve a). Ten minutes after the biasing the coercive field assumes the value of $115 \mathrm{kV} / \mathrm{m}$. The polarization increases rapidly with time at higher fields (cf. curve $b, c$ and $d$ ). The polarization becomes constant at $1300 \mathrm{kV} / \mathrm{m}$ within a few minutes and at $1350 \mathrm{kV} / \mathrm{m}$ as soon as several seconds. Decreasing the field down to $600-500 \mathrm{kV} / \mathrm{m}$ casuses a clear lowering of the hysteresis loop height, and at $E_{0}=480 \mathrm{kV} / \mathrm{m}$ the loop disappears despite the field is much higher than the coercive field ( $E_{0} \equiv E_{3}$ in Fig. 1a).

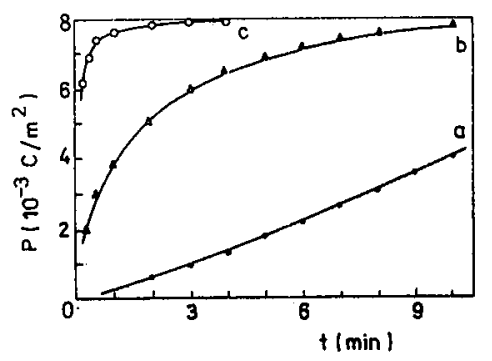

Fig. 3. Temporary changes of the electric polarization at the temperature $3 \mathrm{~K}$ below $T_{c}$. The threshold field $E_{\mathrm{p}}=670 \mathrm{kV} / \mathrm{m}$. The amplitude of $E$ and the coercive field $E_{\mathrm{c}}$ are the following: a) 720,158 ; b) 770,170 ; c) 815,180 .

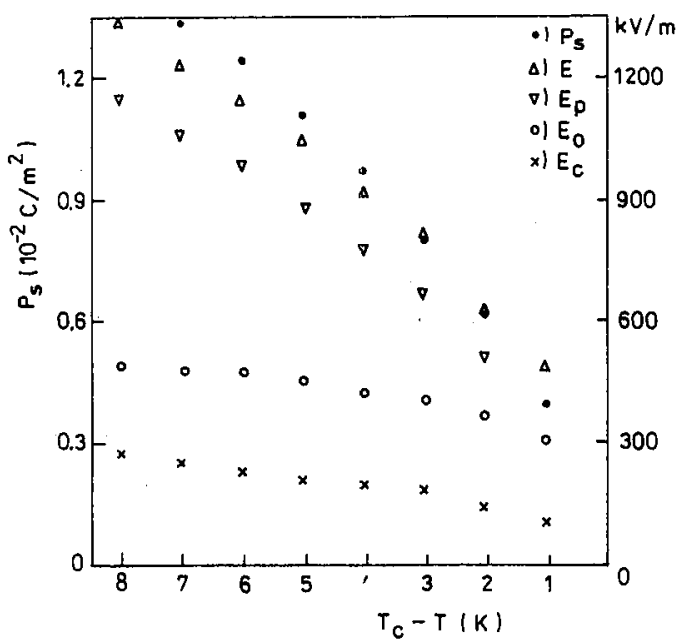

Fig. 4. Temperature dependence of the spontancous polarization $P_{\mathrm{s}}$, the applied electric field $E\left(E \geq E_{2}\right)$, the threshold field $E_{\mathrm{p}}$, the coercive field $E_{\mathrm{c}}$, and the field $E_{0}$ at which the hysteresis loop disappears.

The time dependence of the electric polarization at $6 \mathrm{~K}$ below $T_{\mathrm{c}}$ is similar to that of Fig. 2 with the constant value of the spontaneous polarization being equal to $1.23 \times 10^{-2} \mathrm{C} / \mathrm{m}^{2}$. Figure 3 shows the analogous dependence for $3 \mathrm{~K}$ below $T_{\mathrm{c}}$. Figure 4 shows the temperature dependence of the spontaneous polarization $P_{\mathbf{s}}$ 
along the $c$-axis (with applied fields $E$ at which a constant polarization is acheved within ca. 10 seconds), the applied field $E$, the threshold field $E_{\mathrm{p}}$ required for the hysteresis occurrence and with the field of $E_{0}$ at which the hysteresis disappears. $E_{\mathrm{c}}$ is the coercive field. One may notice that $E_{0}>E_{\mathrm{c}}$. The threshold field depends to some extend upon time lasting from the latest bias process. In order to obtain repeatable results the breaks between the subsequent biases lasted at least two minutes. The hysteresis loop height depends slightly upon the electric bias field. Based on the described observations a slight difference in the spontaneous polarization determined with the Sawyer-Tower circuit and that determined by the pyroelectric method may be expected. The temperature dependence of the pyroelectric coefficient and the spontaneous polarization obtained from the integration are show in Fig. 5. The results of the spontaneous polarization and the electric

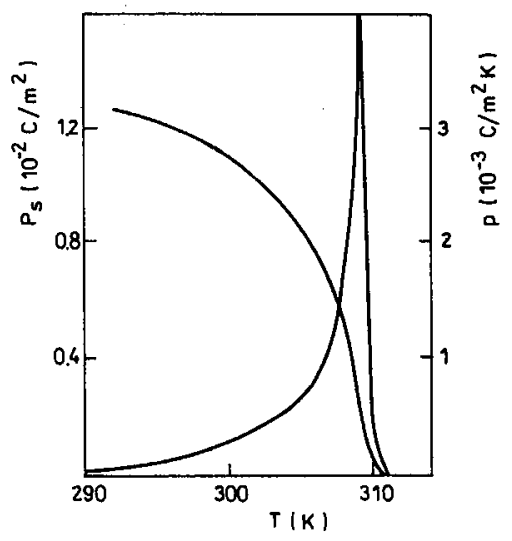

Fig. 5. Temperature dependence of the pyroelectric coefficient and the spontaneous polarization measured on heating. The electrical poling was achieved by cooling a crystal from the paraelectric phase in the field of $300 \mathrm{kV} / \mathrm{m}$.

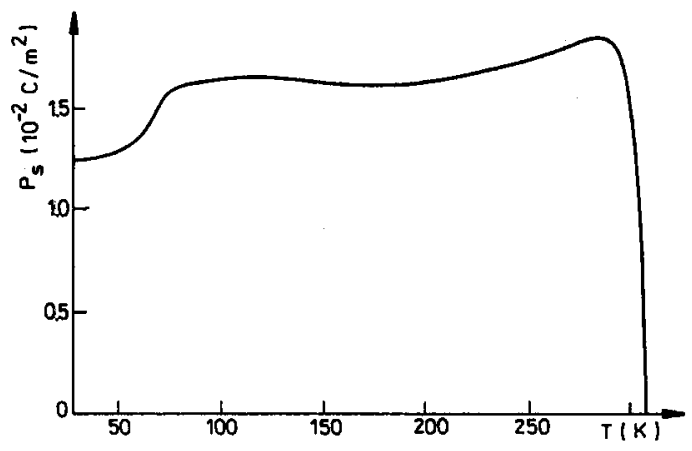

Fig. 6. Temperature dependence of the spontaneous polarization. 


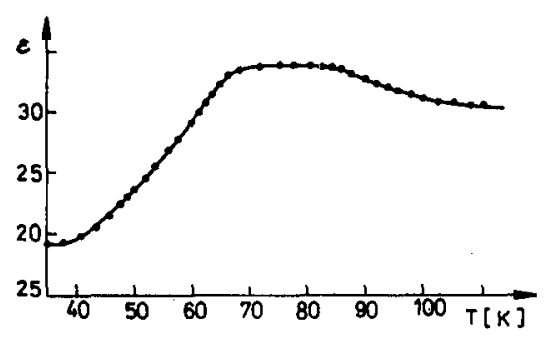

Fig. 7. Temperature dependence of the electric permittivity along the polar $c$-axis in the vicinity of the phase transition temperature $T_{\mathrm{II}}$.

permittivity in the vicinity of the lower phase transition for the crystal grown in subsequent cycles from the same solution are shown in Figs. 6 and 7, respectively, and they clearly differ from those reported in Ref. [10].

The observed difference in the spontaneous polarization shown in Figs. 5 and 6 may have two reasons. The measurements were performed with two different samples of different history and with different electric fields $10^{-3} \mathrm{~V}$ and $1 \mathrm{~V}$, respectively, applied to the samples during the measurement (the measurement methods were different).

\section{Discussion}

For convenience the experimental results for the studied crystals are listed below:

1. Freshly-grown crystals show a non-zero unipolarity.

2. They exhibit a strong internal bias much higher than the coercive fields.

3. Strong alternating electric field can make the crystal younger; the internal field decreases with time and at last a single hysteresis loop occurs.

4. When the electric field applied to the crystal made younger is switched off, the internal field is formed again within a few minutes.

5 . Alternating electric field of $50 \mathrm{~Hz}$ frequency and intensity slightly greater than the coercive field cannot depolarize the crystal.

The field needed to create a single-domain state must be greater than the threshold field.

Hysteresis phenomena similar to those above-mentioned have been observed for $\left[\left(\mathrm{CH}_{3}\right)_{3} \mathrm{NH}\right]_{3} \mathrm{Sb}_{2} \mathrm{Cl}_{9}$ [11]. In many works it is assumed that the internal field is caused by intentional dopants or defects. In our case the crystals were grown under high excess of $\mathrm{HBr}$ and it is likely that $\mathrm{Br}_{2}$ molecules may form defects. Polar properties of the crystal depend upon $\left(\mathrm{CH}_{3} \mathrm{NH}_{3}\right)^{+}$cation ordering [7]. The hysteresis phenomena should then be ascribed to the cations movement due to their free reorientation ability and to their move limitations due to $\mathrm{Br}_{2}$ defects. The cations surrounded by the defects create an internal field. In a weak external one the hysteresis loop does not occur. Whereas a sufficiently strong field causes 
releasing of the cations. Consequences of that are the following: the spontaneous polarization increases and the internal field decreases to zero.

The internal field in the crystal also manifested itself in the electric permittivity measurements. When an external electric field opposite to the internal one was applied to the crystal the electric permittivity was increased with the DC external field up to the moment when the internal field was compensated by the external one. Further bias caused a rapid decrease of the electric permittivity. The crystal cooled down to below $285 \mathrm{~K}$ shows an anomalous decrease in the spontaneous polarization (cf. Fig. 6). The effect is observed both on heating and cooling for all the grown crystals.

At the temperature of $T_{\mathrm{II}}=77 \mathrm{~K}$ the second phase transition is observed. The transition shows an interesting peculiarity since in crystals grown under small excess of $\mathrm{HBr}$ the spontaneous polarization increased on cooling [10] whereas for crystals grown with an additional amount of $\mathrm{HBr}$ in the same solution the polarization and the electric permittivity decreased (cf. Figs. 6 and 7). The reason of that can be ascribed to freezing of a part of cations due to defects after the transition.

Above the temperature of $T_{\mathrm{II}}$ the crystal symmetry is of $P c a 2_{1}$ while a symmetry of the low-temperature phase is unknown. Below $T_{\text {II }}$ spontaneous polarization occurs along the $b$-axis. Taking into account experimental results confirming a continuous character of the phase transition at $T_{\mathrm{II}}$ and basing on the group theory we can conclude that the point group $m$ is the only possible sub-group. Two occurred orientation states in the low-temperature phase refer to a spontaneous deformation. When during this phase transition ferroelastic domains occur, they are expected to decrease both the electric permittivity and the spontaneous polarization in the $c$-axis.

A study of the lower phase transition at $T_{\mathrm{II}}$ along the $b$ - and $a$-axes are in progress and will be published.

\section{References}

[1] R. Jakubas, Solid State Commun. 69, 267 (1989).

[2] R. Matuszewski, R. Jakubas, L. Sobczyk, T. Glowiak, Acta Crystallogr. C 46, 1385 (1990).

[3] R. Cach, R. Jakubas, Ferroelectrics 108, 121 (1990).

[4] C. Pawlaczyk, II. Motsch, R. Jakubas, H.-G. Unruh, Ferroelectrics 108, 127 (1990).

[5] M. Iwata, Y. Ishibashi, J. Phys. Soc. Jpn. 59, 4239 (1990).

[6] A. Miniewicz, R. Jakubas, Ferroelectrics 115, 119 (1991).

[7] R. Jakubas, L. Sobczyk, Ferroelectrics 92, 365 (1989).

[8] B. Kosturek, R. Jakubas, Phys. Status Solidi A 114, K111 (1989).

[9] R. Jakubas, H. Pykacz, Phys. Status Solidi A 115, K17 (1989).

[10] J. Mróz, R. Jakubas, Solid State Commun. 72, 813 (1989).

[11] A. Mikolajczak, R. Cach, Z. Czapla, R. Jakubas, Acta Phys. Pol. A74, 417 (1988). 